\title{
On BL-Algebras and its Interval Counterpart
}

\author{
R. PAIVA ${ }^{1 *}$, R. SANTIAGO ${ }^{2}$ and B. BEDREGAL ${ }^{2}$
}

Received on June 16, 2018 / Accepted on January 22, 2019

\begin{abstract}
Interval Fuzzy Logic and Interval-valued Fuzzy Sets have been widely investigated. Some Fuzzy Logics were algebraically modeled by Peter Hájek as BL-algebras. What is the algebraic counterpart for the interval setting? It is known from the literature that there is an incompatibility between some algebraic structures and its interval counterpart. This paper shows that such incompatibility is also present in the level of BL-algebras. Here we show both: (1) the impossibility of match imprecision and the correctness of the underlying BL-implication and (2) some facts about the intervalization of BL-algebras.
\end{abstract}

Keywords: Fuzzy Logic, BL-Algebras, intervals, correctness principle.

\section{INTRODUCTION}

The motivation of using intervals instead of exact values can also be perceived from the fact that the amount of imprecision can be codified through intervals in terms of its width. Since the operations in $Ł \infty$ are continuous, the resulting interval operations are correct and optimal in the sense of Hickey [10] and Santiago [15], which means that imprecision stored in input intervals are controlled by such operations.

BL-algebras - which were introduced by Hájek [9] - are an algebraic counterpart to Basic Logic (BL) which generalizes the three most commonly used logics in the theory of fuzzy sets; namely: Łukasiewicz logic, product logic and Gödel logic [7,8]. This together with the fact that intervalvalued fuzzy set theory has been revealed as an increasingly promising extension of usual fuzzy sets $[4,5,6,14]$ - namely: the usual membership degrees are replaced by closed intervals in $[0,1]$ - lead us to consider the investigation on the intervalization of BL algebras.

Although BL-algebras has been widely investigated (c.f. $[1,2,3,13,17])$ we found no reference on the literature which takes into account its interval counterpart. Following this standpoint, we

\footnotetext{
*Corresponding author: Rui Paiva - E-mail: rui.brasileiro@ifce.edu.br - https://orcid.org/ 0000-0002-1775-8489

${ }^{1}$ Group for Logic, Language, Information, Theory and Applications (LoLITA), Federal Institute of Education, Science and Technology of Ceará (IFCE), Maracanaú - CE, 61939-140, Brazil. E-mail: rui.brasileiro@ifce.edu.br

${ }^{2}$ Group for Logic, Language, Information, Theory and Applications (LoLITA), Informatics and Applied Mathematics Department, Federal University of Rio Grande do Norte (UFRN), Natal - RN, 59078-970, Brazil. E-mail: regivan@dimap.ufrn.br,bedregal@dimap.ufrn.br
} 
investigate the extension of BL-algebras to interval structures. Namely, by using the notion of abstract intervals and the notion of best interval representation investigated by Santiago et.al. [15], we show how the notion of correctness on intervals affects the algebraic structure of BLalgebras. For example, we prove that there is no best interval representation, $\rightsquigarrow$, for a given BL-algebra implication, $\rightarrow$, (see Theorem 1). Some other properties are also showed.

This paper is organized as follows: Section 1 gives a brief introduction to BL-algebras. Section 2 shows how would be an interval BL-algebra. Section 3 shows the incompatibility between the notions of interval correctness and BL-algebras, provide a way to build an interval-based structure starting from two BL-algebras and give some properties of such a system. Finally, section 4 provides some final remarks.

In this section, we expose a brief introduction of BL-algebras, some of its properties and some examples.

Definition 1. A BL-algebra is a structure $\left\langle L, \wedge, \vee, *, \rightarrow, 0_{L}, 1_{L}\right\rangle$ which satisfies:

(BL1) $\left\langle L, \wedge, \vee, 0_{L}, 1_{L}\right\rangle$ is a bounded lattice with top element $1_{L}$ and bottom element $0_{L}$;

(BL2) $\left\langle L, *, 1_{L}\right\rangle$ is an abelian monoid;

(BL3) The pair $(*, \rightarrow)$ is a Galois connection, i.e: $\forall x, y, z \in L, x * z \leq y$ iff $z \leq x \rightarrow y$;

(BL4) For all $x, y \in L, x *(x \rightarrow y)=x \wedge y$;

(BL5) For all $x, y \in L,(x \rightarrow y) \vee(y \rightarrow x)=1_{L}$.

In what follows we present a list of BL-algebras:

Example 1. The following three structures are important BL-algebra classes.

1. (Algebra of Gödel). This is the algebraic semantics for known Gödel logic, the structure $\langle[0,1], \min , \max , *, \rightarrow, 0,1\rangle$, where $x * y=\min \{x, y\}$ and

$$
x \rightarrow y=1 \text { iff } x \leq y \text { and } y \text { otherwise. }
$$

2. (Algebra of Product). This is the algebraic semantics for known product logic, the structure $\langle[0,1], \min , \max , *, \rightarrow, 0,1\rangle$, where $*$ it is the usual multiplication of real numbers on the unit interval $[0,1]$ and

$$
x \rightarrow y=1 \text { iff } x \leq y \text { and } y / x \text { otherwise. }
$$

3. (Algebra of Łukasiewicz). This is the algebraic semantics for known Łukasiewicz logic, the structure $\langle[0,1], \min , \max , *, \rightarrow, 0,1\rangle$, where $x * y=\max \{0, x+y-1\}$ and

$$
x \rightarrow y=1 \text { iff } x \leq y \text { and } \min \{1,1-x+y\} \text { otherwise. }
$$


Example 2. Let $X$ be a nonempty set and let $\mathscr{P}(X)$ be the family of all subsets of $X$. Define operations $*$ and $\rightarrow$ by

$$
A * B=A \cap B \text { and } A \rightarrow B=A^{c} \cup B
$$

for all $A, B \in \mathscr{P}(X)$, respectively. Then $\langle\mathscr{P}(X), \cap, \cup, *, \rightarrow, \emptyset, X\rangle$ is a BL-algebra. We call $\mathscr{P}(X)$ as the power BL-algebra of $X$.

Example 3. If $\langle L, \wedge, \vee, *, \rightarrow, 0,1\rangle$ is a BL-algebra and $X$ is a nonempty set, then the functions space $L^{X}$ becomes a $B L$-algebra $\left\langle L^{X}, \wedge, \vee, *, \rightarrow, \overline{0}, \overline{1}\right\rangle$ with the operations are defined pointwise. If $f, g \in L^{X}$, then

$$
\begin{aligned}
(f \wedge g)(x) & =f(x) \wedge g(x) \\
(f \vee g)(x) & =f(x) \vee g(x) \\
(f * g)(x) & =f(x) * g(x) \\
(f \rightarrow g)(x) & =f(x) \rightarrow g(x)
\end{aligned}
$$

for all $x, y \in X$ and $\overline{0}, \overline{1}: X \rightarrow L$ are the constant functions associated with $0,1 \in L$.

Proposition 1. (see [9]) If $\left\langle L, \wedge, \vee, *, \rightarrow, 0_{L}, 1_{L}\right\rangle$ is a BL-algebra and $x, y, z \in L$ then:

(BL6) $x *(x \rightarrow y) \leq y$

(BL7) If $x \leq y$ then $x * z \leq y * z$

(BL8) $x * 0_{L}=0_{L}$

(BL9) If $x \leq y$ then $y \rightarrow z \leq x \rightarrow z$ (First Place Antitonicity - FPA)

(BL10) If $x \leq y$ then $z \rightarrow x \leq z \rightarrow y$ (Second Place Isotonicity - SPI)

(BL11) $x \leq y$ iff $x \rightarrow y=1_{L}($ Order Property - OP)

(BL12) $x \rightarrow(y \rightarrow z)=y \rightarrow(x \rightarrow z)$ (Exchange Principle - EP)

(BL13) $(x \vee y) * z=(x * z) \vee(y * z)$

$($ BL14) $(x \rightarrow y) *(y \rightarrow z) \leq x \rightarrow z$

(BL15) $x \rightarrow y \leq(x * z) \rightarrow(z * y)$

(BL16) $(x \wedge y) \rightarrow x=1_{L}$

(BL17) $(x \rightarrow y)=x \rightarrow(x \wedge y)$

(BL18) $(x * z) \rightarrow y=z \rightarrow(x \rightarrow y)$

(BL19) $x \leq y \rightarrow(x * y)$ 
Some of the above examples have elements which are not finitely representable. That is, they are algebras which contain irrational numbers, like $\pi-3 \in[0,1]$. A question posed is how can we represent the underlying imprecision of such structures? One answer for that is the application of Interval Mathematics, which model the imprecision in numerical calculations ( [11], [12]) and provides algorithms with rigorous control of errors. In the next section, we show that this approach induces algebraic structures which cannot be BL-algebras. Hence, a new algebraic structure will be revealed in order to obtain a suitable interval counterpart of a BL-algebra.

\section{INTERVAL BL-ALGEBRAS}

In what follows we introduce some required concepts, like the abstract notion of intervals. The aim is to provide the ability to use intervals to represent the elements of a BL-algebra $\left\langle L, \wedge, \vee, *, \rightarrow, 0_{L}, 1_{L}\right\rangle$.

Definition 2. (Abstract interval) Given a poset $\langle L, \leq\rangle$, the set $[a, b]=\{x \in L \mid a \leq x \leq b\}$ is called the closed interval with endpoints $a$ and $b$. The set $I(L)=\{[a, b] \mid a \leq b$ and $a, b \in L\}$ is the set of all interval of elements in $L$. For any $X=[\underline{X}, \bar{X}] \in I(L), \underline{X}$ is called the lower bound of $X$ and $\bar{X}$ is called the upper bound of $X$. When $\underline{X}=\bar{X}$, the interval $X$ it called degenerated. The embedding $i: L \rightarrow I(L)$ such that $i(x)=[x, x]$ is called natural embedding.

We also define a partial order on $I(L)$ called Kulisch-Miranker order: For all $X, Y \in I(L)$,

$$
X \sqsubseteq Y \Leftrightarrow \underline{X} \leq \underline{Y} \text { and } \bar{X} \leq \bar{Y} .
$$

The fundamental property of interval mathematics is the notion of interval correctness. It was studied by Santiago et $\mathrm{al}^{1}$ [15]. Instead of correctness the authors used the term representation. Essentially, correctness or representation means that if $F$ is correct with respect to $f$, then we can enfold any exact value $r$ in a closed interval $[a, b]$ and then simply operate with such "envelopes" by using $F$, because the resulting interval $F([a, b])$ will enfold the desired result $f(r)$, in symbols: $r \in[a, b] \Rightarrow f(r) \in F([a, b])$. In what follows we show this notion for binary operations: a binary interval operation $\downarrow$ defined on $I(L)$ represents a binary operation $\diamond$ defined on $L$ whenever,

$$
(x, y) \in[a, b] \times[c, d] \text { implies } x \diamond y \in[a, b] \diamond[c, d] .
$$

Example 4 (Arithmetic operations on real intervals). Let $[a, b]$ and $[c, d]$ be real intervals. The interval operations of sum, difference and product are defined in the following way:

(i) $[a, b] \oplus[c, d]=[a+c, b+d]$,

(ii) $[a, b] \ominus[c, d]=[a-d, b-c]$,

\footnotetext{
${ }^{1}$ In this paper the authors use the term representation instead of correctness because interval expressions could be faced not just as machine representations of an exact calculation, but also as an instance of mathematical representation of real numbers.
} 
(iii) $[a, b] \otimes[c, d]=[\min P, \max P]$, where $P=\{a \times c, a \times d, b \times c, b \times d\}$.

Notice that for each interval operation $\bullet \in\{\oplus, \ominus, \otimes\}$ and their respective elementary real operation $\circ \in\{+,-, \times\}$ it follows that $[a, b] \bullet[c, d]=\{\alpha \circ \beta \mid \alpha \in[a, b]$ and $\beta \in[c, d]\}$. Therefore, in each case, the binary interval operation $\bullet$ defined on $I(\mathbb{R})$ represents a binary operation $\circ$ defined on $\mathbb{R}$. The following example ratifies the thesis that not all extension of arithmetic operations for intervals is correct.

Example 5. Given two intervals $X=[\underline{X}, \bar{X}]$ and $Y=[\underline{Y}, \bar{Y}]$, the interval $X \ominus Y$ defined by

$$
X \ominus Y=[\min \{\underline{X}-\underline{Y}, \bar{X}-\bar{Y}\}, \max \{\underline{X}-\underline{Y}, \bar{X}-\bar{Y}\}]
$$

extends the subtraction on real numbers. Notice that $[4,5]-[4,5]=[0,0]$, however the rational numbers 4.7 and 4.1 belong to the interval $[4,5]$, but $4.7-4.1=0.6 \notin[0,0]$. Thus, the interval binary operator $\ominus$ is not correct with respect to binary real operator “-”.

Another desirable property according to Hickey [10] is Optimality: The resulting interval should be the smallest possible which satisfy the correctness criterion. The process of giving the correct and optimal interval version $F$ for a function $f$ is called: "intervalization".

Since BL-algebras are partially ordered systems $\langle L, \leq\rangle$ it is possible to apply Definition 2 to obtain the partial order $\langle I(L), \sqsubseteq\rangle$. The question is: From this partial order is it possible to define a BL-algebra which represents $L$ ? The following propositions will show that the answer is negative.

For now, observe that it is possible to obtain a BL-algebra of intervals from some BL-algebras.

Definition 3. Given a BL-algebra: $\left\langle L, \wedge, \vee, *, \rightarrow, 0_{L}, 1_{L}\right\rangle$ in which $L$ is a complete lattice, we define the following binary operations on $I(L)$ :

(1) $[a, b] \sqcap[c, d]=[a \wedge c, b \wedge d]$,

(2) $[a, b] \sqcup[c, d]=[a \vee c, b \vee d]$,

(3) $[a, b] \circledast[c, d]=[a * c, b * d]$,

(4) $[a, b] \Rightarrow[c, d]=\bigsqcup\{[e, f] \in I(L) \mid[a, b] \circledast[e, f] \sqsubseteq[c, d]\}$.

Proposition 2. The structure $\langle I(L), \sqcap, \sqcup, \mathbf{0}, \mathbf{1}\rangle$ it is a complete lattice with the top element $\mathbf{1}=$ $\left[1_{L}, 1_{L}\right]$ and the bottom element $\mathbf{0}=\left[0_{L}, 0_{L}\right]$.

Proof. According to the definition of $\sqcap$ and $\sqcup$ operators, just consider for each $[a, b],[c, d] \in I(L)$, $\Pi\{[a, b],[c, d]\}=[a, b] \sqcap[c, d]$ and $\bigsqcup\{[a, b],[c, d]\}=[a, b] \sqcup[c, d]$. Thus $\left\langle I(L), \sqcap, \sqcup, 0_{L}, 1_{L}\right\rangle$ is a lattice. Now consider the non-empty set $X \subseteq I(L)$. It is obvious that $\left[0_{L}, 0_{L}\right]$ is a lower bound of $X$, then the set:

$$
X^{\ell}=\{J \in I(L) \mid J \text { is lower bound of } X\}
$$


it is not empty. Define

$$
v=\bigvee_{A \in X^{\ell}}(\underline{A}) \quad \text { and } \quad w=\bigvee_{A \in X^{\ell}}(\bar{A}) .
$$

This implies that $[v, w]$ is lower bound of $X$. We affirm that $[v, w]$ it is the largest of the lower bounds of $X$. Indeed, suppose there exists $[r, s] \in X^{\ell}$ such that $[v, w] \sqsubseteq[r, s]$, then $v \leq r$ and $w \leq s$. On the other hand, by way $v$ and $w$ are defined, we have $v \geq r$ and $w \geq s$. Therefore $v=r, w=s$ and hence $\prod X=[v, w]$. Similarly, since $\left[1_{L}, 1_{L}\right]$ is upper bound for $X$, define

$$
X^{u}=\{T \in I(L) \mid T \text { is upper bound of } X\} \neq \emptyset .
$$

and call

$$
m=\bigwedge_{B \in X^{u}}(\underline{B}) \quad \text { and } \quad n=\bigwedge_{B \in X^{u}}(\bar{B}) .
$$

This leads us to conclude that $\bigsqcup X=[m, n]$.

Proposition 3. $\langle I(L), \circledast, \mathbf{1}\rangle$ is an Abelian monoid with identity $\mathbf{1}=\left[1_{L}, 1_{L}\right]$.

Proof. Since that the operator $*$ is associative, commutative and has identity $1_{L}$, just check these properties for $\circledast$ operator what is straightforward.

Proposition 4. $\langle I(L), \sqcap, \sqcup, \circledast, \Rightarrow, \mathbf{0}, \mathbf{1}\rangle$ is a BL-algebra.

Proof. Notice that the axioms (BL1) and (BL2) follow, respectively, from propositions 2 and 3. Moreover, given $X, Y, Z \in I(L)$,

$$
Z \sqsubseteq(X \Rightarrow Y) \text { if and only if }[\underline{Z}, \bar{Z}] \sqsubseteq[\underline{X} \rightarrow \underline{Y}, \bar{X} \rightarrow \bar{Y}] .
$$

In fact,

$$
\begin{aligned}
& Z \sqsubseteq(X \Rightarrow Y) \quad \text { iff } \quad Z \sqsubseteq \bigsqcup\{W \mid X \circledast W \sqsubseteq Y\} \\
& \text { iff } \quad \underline{Z} \leq \bigsqcup\{W \mid X \circledast W \sqsubseteq Y\} \text { and } \bar{Z} \leq \overline{\bigsqcup\{W \mid X \circledast W \sqsubseteq Y\}} \\
& \text { iff } \quad \underline{Z} \leq \bigvee\{\underline{W} \mid X \circledast W \sqsubseteq Y, \text { for some } W\} \text { and } \bar{Z} \leq \bigvee\{\bar{W} \mid X \circledast W \sqsubseteq Y, \text { for some } W\} .
\end{aligned}
$$

However,

$$
\begin{aligned}
\bigvee\{\underline{W} \mid X \circledast W \sqsubseteq Y, \text { for some } W\} & =\bigvee\{\underline{W} \mid \underline{X \circledast W} \leq \underline{Y} \text { and } \overline{X \circledast W} \leq \bar{Y}, \text { for some } W\} \\
& =\bigvee\{\underline{W} \mid \underline{X} * \underline{W} \leq \underline{Y} \text { and } \bar{X} * \bar{W} \leq \bar{Y}, \text { for some } W\} \\
& \leq \bigvee\{\underline{W} \mid \underline{X} * \underline{W} \leq \underline{Y}\} .{ }^{2}
\end{aligned}
$$

Analogously,

$$
\begin{aligned}
\bigvee\{\bar{W} \mid X \circledast W \sqsubseteq Y, \text { for some } W\} & =\bigvee\{\bar{W} \mid \underline{X \circledast W} \leq \underline{Y} \text { and } \overline{X \circledast W} \subseteq \bar{Y}, \text { for some } W\} \\
& =\bigvee\{\bar{W} \mid \underline{X} * \underline{W} \leq \underline{Y} \text { and } \bar{X} * \bar{W} \leq \bar{Y}, \text { for some } W\} \\
& \leq \bigvee\{\bar{W} \mid \bar{X} * \bar{W} \leq \bar{Y}\} .
\end{aligned}
$$

${ }^{2} A \subseteq B$ implies $\bigvee A \leq \bigvee B$, since both exist. 
This means that $\underline{Z} \leq \underline{X} \rightarrow \underline{Y}$ and $\bar{Z} \leq \bar{X} \rightarrow \bar{Y}$. From such inequalities we can establish the following

$$
\begin{array}{rll}
Z \sqsubseteq(X \Rightarrow Y) \quad \text { iff } & \underline{Z} \leq \underline{X} \rightarrow \underline{Y} \text { and } \bar{Z} \leq \bar{X} \rightarrow \bar{Y} \\
& \text { iff } \quad \underline{X} * \underline{Z} \leq \underline{Y} \text { and } \bar{X} * \bar{Z} \leq \bar{Y} \\
& \text { iff } \quad[\underline{X} * \underline{Z}, \bar{X} * \bar{Z}] \sqsubseteq[\underline{Y}, \bar{Y}] \\
& \text { iff } \quad X \circledast Z \sqsubseteq Y .
\end{array}
$$

Therefore, for all $X, Y, Z \in I(L)$ the pair $(\circledast, \Rightarrow)$ is Galois connection, hence (BL3) is satisfied. Now let's check out the Axiom (BL4). Indeed, for all $X, Y \in I(L)$,

$$
\begin{aligned}
& X \circledast(X \Rightarrow Y) \quad=\quad[\underline{X}, \bar{X}] \circledast([\underline{X}, \bar{X}] \Rightarrow[\underline{Y}, \bar{Y}]) \\
& =\quad[\underline{X}, \bar{X}] \circledast(\bigsqcup\{[\underline{Z}, \bar{Z}] \in I(L) \mid[\underline{X}, \bar{X}] \circledast[\underline{Z}, \bar{Z}] \sqsubseteq[\underline{Y}, \bar{Y}]\}) \\
& \text { Eq.(2.1) } \quad[\underline{X}, \bar{X}] \circledast[\underline{X} \rightarrow \underline{Y}, \bar{X} \rightarrow \bar{Y}] \\
& =[\underline{X} *(\underline{X} \rightarrow \underline{Y}), \bar{X} *(\bar{X} \rightarrow \bar{Y})] \\
& =\quad[\underline{X} \wedge \underline{Y}, \bar{X} \wedge \bar{Y}] \\
& =\quad[\underline{X}, \bar{X}] \sqcap[\underline{Y}, \bar{Y}] \\
& =\quad X \sqcap Y \text {. }
\end{aligned}
$$

Finally, for the axiom (BL5), we can simplify writing, for all $X, Y \in I(L)$

$$
\begin{aligned}
(X \Rightarrow Y) \sqcup(Y \Rightarrow X) & =(\bigsqcup\{Z \in I(L) \mid X \circledast Z \sqsubseteq Y\}) \sqcup(\bigsqcup\{Z \in I(L) \mid Y \circledast Z \leq X\}) \\
& =[\underline{X} \rightarrow \underline{Y}, \bar{X} \rightarrow \bar{Y}] \sqcup[\underline{Y} \rightarrow \underline{X}, \bar{Y} \rightarrow \bar{X}] \\
& =[(\underline{X} \rightarrow \underline{Y}) \vee(\underline{Y} \rightarrow \underline{X}),(\bar{X} \rightarrow \bar{Y}) \vee(\bar{Y} \rightarrow \bar{X})] \\
& =\left[1_{L}, 1_{L}\right] \\
& =\mathbf{1} .
\end{aligned}
$$

This completes the proof.

Although it is possible to obtain interval BL-algebras from BL-algebras, the next theorem shows that none of them will provide correct implications. This is informally stated in [16].

Theorem 1. Given a BL-algebra $\left\langle L, \wedge, \vee, *, \rightarrow, 0_{L}, 1_{L}\right\rangle$ there is no interval binary operator $\rightsquigarrow$ correct with respect to binary operator $\rightarrow$ such that $\langle I(L), \sqcap, \sqcup, \circledast, \rightsquigarrow, \mathbf{0}, \mathbf{1}\rangle$ is an BL-algebra.

Proof. Let $a, b \in L$ distinct. There are two cases to consider: $a$ and $b$ are comparable or not. If $a$ and $b$ are comparable, assumes without loss of generality that $a \leq b$, therefore $a \rightarrow b=1_{L}$. Therefore $b \rightarrow a \neq 1_{L}$, since otherwise we would have $b \leq a$, which contradicts the fact that $a$ and $b$ are distinct. So we have $(b, a) \in[a, b] \times[a, b]$ but $b \rightarrow a \notin([a, b] \rightsquigarrow[a, b])=\left[1_{L}, 1_{L}\right]$. In contrast, if $a$ and $b$ are incomparable, once $L$ is a bounded lattice, the inequalities $\bigwedge\{a, b\} \leq a \leq \bigvee\{a, b\}$ and $\bigwedge\{a, b\} \leq b \leq \bigvee\{a, b\}$ 
are valid. Thus $(a, b) \in[\bigwedge\{a, b\}, \bigvee\{a, b\}] \times[\bigwedge\{a, b\}, \bigvee\{a, b\}]$, however $a \rightarrow b \neq 1_{L}$ and $[\bigwedge\{a, b\}, \bigvee\{a, b\}] \rightsquigarrow[\bigwedge\{a, b\}, \bigvee\{a, b\}]=\left[1_{L}, 1_{L}\right]$. Therefore, in both cases the interval binary operator $\rightsquigarrow$ is not correct with respect to binary operator $\rightarrow$.

\section{THE BEST INTERVAL REPRESENTATION}

The most important property of Moore Interval arithmetic [12] is not just its correctness, i.e, $x \in[a, b] \wedge y \in[c, d] \Rightarrow x+y \in[a, b]+[c, d]$, but its optimality; namely $[a, b]+[c, d]$ is the tightest interval contaning $x+y$. Santiago et al [15] call this feature as: "The best interval representation" of "+". More generally:

Definition 4. Given a lattice $\langle L, \wedge, \vee\rangle$, an interval operator $\Delta: I(L) \times I(L) \rightarrow I(L)$ is representable if there exist operators, $\Delta_{1}, \Delta_{2}: L \times L \rightarrow L$ such that for each $X, Y \in I(L)$, with $\alpha \in X$ and $\beta \in Y$, we have that

$$
\Delta(X, Y)=\left[\inf \Delta_{1}(\alpha, \beta) \cup \Delta_{2}(\alpha, \beta), \sup \Delta_{1}(\alpha, \beta) \cup \Delta_{2}(\alpha, \beta)\right]
$$

In this case $\Delta_{1}$ and $\Delta_{2}$ are called representants of $\Delta$ and $\Delta$ is the best interval representation of $\Delta_{1}$ and $\Delta_{2}$. We use the notation $\widehat{\Delta_{1,2}}$ for the interval operator which have $\Delta_{1}$ and $\Delta_{2}$ as their representants. Observe that case for some $X, Y \in I(L), \Delta_{1}(\alpha, \beta) \cup \Delta_{2}(\alpha, \beta)$ has not an infimum or supremum then they are not representant of $\Delta$.

Proposition 5. If $\left\langle L, \wedge, \vee, *_{1}, \rightarrow_{1}, 0_{L}, 1_{L}\right\rangle$ and $\left\langle L, \wedge, \vee, *_{2}, \rightarrow_{2}, 0_{L}, 1_{L}\right\rangle$ are BL-algebras such that $*_{1} \leq *_{2}$ and $\rightarrow_{2} \leq \rightarrow_{1}$, then the functions $*, \rightarrow: I(L) \times I(L) \rightarrow I(L)$ defined by

(i) $[a, b] *[c, d]=\left[a *_{1} c, b *_{2} d\right]$;

(ii) $[a, b] \rightarrow[c, d]=\left[b \rightarrow_{2} c, a \rightarrow_{1} d\right]$,

are representable with $*_{1}$ and $*_{2}$, and, $\rightarrow_{1}$ and $\rightarrow_{2}$, as representants, respectively. Therefore, $*=\widehat{*_{1,2}}$ and $\rightarrow=\widehat{\rightarrow_{1,2}}$.

Proof. Let be $\gamma \in \Delta_{1}(\alpha, \beta) \cup \Delta_{2}(\alpha, \beta)$, i.e, $\gamma \in L$ such that $\gamma=\Delta_{1}(\alpha, \beta)$ or $\gamma=\Delta_{2}(\alpha, \beta)$. We must show that:

(i) $\inf \Delta_{1}(\alpha, \beta) \cup \Delta_{2}(\alpha, \beta)=a *_{1} c$ and $\sup \Delta_{1}(\alpha, \beta) \cup \Delta_{2}(\alpha, \beta)=b *_{2} d$. Since that $\Delta_{i}(\alpha, \beta)=$ $\alpha *_{i} \beta$ with $i \in\{1,2\}$, we affirm that $a *_{1} c$ and $b *_{2} d$ are, respectively, a lower bound and an upper bound of the set in question. In fact, let be $\alpha \in[a, b]$ and $\beta \in[c, d]$, i.e, $a \leq \alpha \leq b$ and $c \leq \beta \leq d$. Then, for all $x \in[c, d]$ and for all $y \in[a, b]$, we have:

$$
a *_{1} x \leq \alpha *_{1} x \leq b *_{1} x \quad \text { and } \quad c *_{2} y \leq y *_{2} \beta \leq y *_{2} d .
$$

In particular, when $x=c$ and $y=b$, we have:

$$
a *_{1} c \leq \alpha *_{1} c \leq b *_{1} c \quad \text { and } \quad c *_{2} b \leq b *_{2} \beta \leq b *_{2} d .
$$


Using the fact that $*_{1} \leq *_{2}$, we have the chain:

$$
a *_{1} c \leq \alpha *_{1} \beta \leq \alpha *_{2} \beta \leq b *_{2} d .
$$

Therefore, $a *_{1} c \leq \gamma \leq b *_{2} d$. On the other hand, if there are $u, v \in L$ such that $u \leq \gamma \leq v$, namely, $u \leq \alpha *_{1} \beta \leq \alpha *_{2} \beta \leq v$, for all $\alpha \in[a, b]$ and for all $\beta \in[c, d]$. Then, in particular, $u \leq a *_{1} c$ and $b *_{2} d \leq v$, which shows that $a *_{1} c$ and $b *_{2} d$ are, respectively, greatest lower bound and lowest upper bound of the said set.

(ii) inf $\Delta_{1}(\alpha, \beta) \cup \Delta_{2}(\alpha, \beta)=b \rightarrow_{2} c$ and $\sup \Delta_{1}(\alpha, \beta) \cup \Delta_{2}(\alpha, \beta)=a \rightarrow_{1} d$. Similarly to the previous item, since $\Delta_{i}(\alpha, \beta)=\alpha \rightarrow_{i} \beta$ with $i \in\{1,2\}$, we can obtain the following inequalities:

$$
b \rightarrow_{2} c \leq \alpha \rightarrow_{2} c \leq a \rightarrow_{2} c \quad \text { and } \quad a \rightarrow_{1} c \leq a \rightarrow_{1} \beta \leq a \rightarrow_{1} d
$$

Using the fact that $\rightarrow_{2} \leq \rightarrow 1$ we have the chain

$$
b \rightarrow_{2} c \leq \alpha \rightarrow_{2} \beta \leq a \rightarrow_{2} c \leq a \rightarrow_{1} c \leq \alpha \rightarrow_{1} \beta \leq a \rightarrow_{1} d
$$

which allows us to complete the desired result.

In particular, when the operators $\Delta_{1}$ and $\Delta_{2}$ coincide, we have the following:

Corollary 1. If $\left\langle L, \wedge, \vee, *, \rightarrow, 0_{L}, 1_{L}\right\rangle$ is a BL-algebra then the following items provide the best interval representation of their corresponding operators:

(i) $[a, b] \widehat{\wedge}[c, d]=[a \wedge c, b \wedge d]$;

(ii) $[a, b] \widehat{\vee}[c, d]=[a \vee c, b \vee d]$;

(iii) $[a, b] \widehat{*}[c, d]=[a * c, b * d]$;

(iv) $[a, b] \widehat{\rightarrow}[c, d]=[b \rightarrow c, a \rightarrow d]$.

In the following, we provide some results of intervalization of BL-algebras.

Theorem 2. Let $\left\langle L, \wedge, \vee, *_{1}, \rightarrow_{1}, 0_{L}, 1_{L}\right\rangle$ and $\left\langle L, \wedge, \vee, *_{2}, \rightarrow_{2}, 0_{L}, 1_{L}\right\rangle$ be BL-algebras such that $*_{1} \leq *_{2}$ and $\rightarrow_{2} \leq \rightarrow_{1}$. Then, for all $X, Y, Z \in I(L)$, the following properties remain valid:

(A-1) If $X \sqsubseteq Y$ then $X \widehat{*_{1,2}} Z \sqsubseteq Y \widehat{* 1,2} Z$;

$(A-2) X \widehat{*_{1,2}} \mathbf{0}=\mathbf{0}$, where $\mathbf{0}=\left[0_{L}, 0_{L}\right]$;

(A-3) If $Y \sqsubseteq Z$ then $X \widehat{\rightarrow 1,2} Y \sqsubseteq \widehat{\rightarrow 1,2} Z$ (Second Place Isotonicity);

$\left.(A-4) X \widehat{\rightarrow_{1,2}}(Y \widehat{\rightarrow 1,2} Z)=Y \widehat{\rightarrow 1,2}_{1, \widehat{\rightarrow_{1,2}}} Z\right)$ (Exchange Principle);

(A-5) If $X \sqsubseteq Y$ then $Y \widehat{\rightarrow 1,2} Z \sqsubseteq X \widehat{11,2} Z$ (First Place Antitonicity); 
$(A-6)(X \widehat{\vee} Y) \widehat{*_{1,2}} Z=\left(X \widehat{*_{1,2}} Z\right) \widehat{\vee}\left(Y \widehat{*_{1,2}} Z\right)$;

$(A-7)\left(X \widehat{*_{1,2}} Z\right) \widehat{\rightarrow_{1,2}} Y=Z \widehat{\rightarrow_{1,2}}\left(X \widehat{\rightarrow_{1,2}} Y\right)$.

Proof. The properties of operator presented in Section 1 are related with the respective properties of the best interval operator enrolled above.

(A-1): $X \sqsubseteq Y \Leftrightarrow[\underline{X}, \bar{X}] \sqsubseteq[\underline{Y}, \bar{Y}] \Leftrightarrow \underline{X} \leq \underline{Y}$ and $\bar{X} \leq \bar{Y}$. Hence by (BL7) we have $\underline{X} *_{1} \underline{Z} \leq \underline{Y} *_{1} \underline{Z}$ and $\bar{X} *_{2} \bar{Z} \leq \bar{Y} *_{2} \bar{Z}$ and, therefore, $\left[\underline{X} *_{1} \underline{Z}, \bar{X} *_{2} \bar{Z}\right] \sqsubseteq\left[\underline{Y} *_{1} \underline{Z}, \bar{Y} *_{2} \bar{Z}\right] \Leftrightarrow X \widehat{*_{1,2}} Z \sqsubseteq Y *_{1,2} Z$.

(A-2): Since $\mathbf{0}=\left[0_{L}, 0_{L}\right]$ we have

$$
\begin{array}{rll}
X \widehat{*} \mathbf{0} & = & {[\underline{X}, \bar{X}] \widehat{*}\left[0_{L}, 0_{L}\right]} \\
& = & {\left[\underline{X} *{ }_{1} 0_{L}, \bar{X} *_{2} 0_{L}\right]} \\
& \stackrel{(B L 8)}{=} & {\left[0_{L}, 0_{L}\right]} \\
= & \mathbf{0} .
\end{array}
$$

(A-3): Let $X, Y, Z \in I(L)$ such that $Y \sqsubseteq Z$. Since $\underline{Y} \leq \underline{Z}, \bar{Y} \leq \bar{Z}$ and $\rightarrow_{i}$, with $i \in\{1,2\}$, satisfy Property (BL10), then it holds that $\bar{X} \rightarrow_{2} \underline{Y} \leq \bar{X} \rightarrow_{2} \underline{Z}$ and $\underline{X} \rightarrow_{1} \bar{Y} \leq \underline{X} \rightarrow_{1} \bar{Z}$. So follows that $X \widehat{\rightarrow_{1,2}} Y \sqsubseteq X \widehat{\rightarrow_{1,2}} Z$.

(A-4) By Property (BL12), it follows that

$$
\begin{aligned}
X \widehat{\rightarrow 1,2}_{\left.1, \widehat{\rightarrow_{1,2}} Z\right)} & =X \widehat{\rightarrow}_{1,2}\left(\left[\bar{Y} \rightarrow_{2} \underline{Z}, \underline{Y} \rightarrow_{1} \bar{Z}\right]\right) \\
& =\left[\bar{X} \rightarrow_{2}\left(\bar{Y} \rightarrow_{2} \underline{Z}\right), \underline{X} \rightarrow_{1}\left(\underline{Y} \rightarrow_{1} \bar{Z}\right)\right] \\
& =\left[\bar{Y} \rightarrow_{2}\left(\bar{X} \rightarrow_{2} \underline{Z}\right), \underline{Y} \rightarrow_{1}\left(\underline{X} \rightarrow_{1} \bar{Z}\right)\right] \\
& =Y \widehat{\rightarrow 1}_{1,2}\left(\left[\bar{X} \rightarrow_{1} \underline{Z}, \underline{X} \rightarrow_{2} \bar{Z}\right]\right) \\
& =Y \widehat{\rightarrow 1}_{1,2}\left(X \widehat{\rightarrow}_{1,2} Z\right) .
\end{aligned}
$$

(A-5): Let $X, Y, Z \in I(L)$ such that $X \sqsubseteq Y$. Since $\underline{X} \leq \underline{Y}, \bar{X} \leq \bar{Y}$ and $\rightarrow_{i}$, with $i \in\{1,2\}$ satisfy Property (BL9), then it holds that $\bar{X} \rightarrow_{2} \underline{Z} \geq \bar{Y} \rightarrow_{2} \underline{Z}$ and $\underline{X} \rightarrow_{1} \bar{Z} \geq \underline{Y} \rightarrow_{1} \bar{Z}$. So follows that $Y \widehat{\rightarrow 1,2}_{1, X} \widehat{\rightarrow 1}_{1,2} Z$.

(A-6): Indeed,

$$
\begin{aligned}
& (X \widehat{\vee} Y) \widehat{* 1,2} Z \quad=\quad[\underline{X} \vee \underline{Y}, \bar{X} \vee \bar{Y}] \widehat{* 1,2}[\underline{Z}, \bar{Z}] \\
& =\left[(\underline{X} \vee \underline{Y}) *_{1} \underline{Z},(\bar{X} \vee \bar{Y}) *_{2} \bar{Z}\right] \\
& \stackrel{(B L 13)}{=}\left[\left(\underline{X} *{ }_{1} \underline{Z}\right) \vee\left(\underline{Y} *{ }_{1} \underline{Z}\right),\left(\bar{X} *_{2} \bar{Z}\right) \vee\left(\bar{Y} *_{2} \bar{Z}\right)\right] \\
& =\left[\underline{X} *_{1} \underline{Z}, \bar{X} *_{2} \bar{Z}\right] \widehat{\vee}\left[\underline{Y} *_{1} \underline{Z}, \bar{Y} *_{2} \bar{Z}\right] \\
& =(X \widehat{* 1,2} Z) \widehat{\nabla}(Y \widehat{* 1,2} Z) \text {. }
\end{aligned}
$$


(A-7): The result follows directly developing both sides of equality. Let's see:

$$
\begin{array}{rll}
\left(X \widehat{*_{1,2}} Z\right) \widehat{\rightarrow_{1,2}} Y & = & \left([\underline{X}, \bar{X}] \widehat{*_{1,2}}[\underline{Z}, \bar{Z}]\right) \widehat{\rightarrow_{1,2}}[\underline{Y}, \bar{Y}] \\
& = & \left(\left[\underline{X} *_{1} \underline{Z}, \bar{X} *_{2} \bar{Z}\right] \widehat{\rightarrow_{1,2}}[\underline{Y}, \bar{Y}]\right. \\
& = & {\left[\left(\bar{X} *_{2} \bar{Z}\right) \rightarrow_{2} \underline{Y},\left(\underline{X} *_{1} \underline{Z}\right) \rightarrow_{1} \bar{Y}\right]} \\
& \stackrel{(B L 18))}{=} & {\left[\bar{Z} \rightarrow_{2}\left(\bar{X} \rightarrow_{2} \underline{Y}\right), \underline{Z} \rightarrow_{1}\left(\underline{X} \rightarrow_{1} \bar{Y}\right)\right] .}
\end{array}
$$

Similarly

$$
\begin{aligned}
Z \widehat{\rightarrow 1,2}_{1, \widehat{\rightarrow_{1,2}} Y}(X) & {[\underline{Z}, \bar{Z}] \widehat{\rightarrow_{1,2}}\left([\underline{X}, \bar{X}] \widehat{\rightarrow_{1,2}}[\underline{Y}, \bar{Y}]\right) } \\
& =[\underline{Z}, \bar{Z}] \widehat{\rightarrow}_{1,2}\left(\left[\bar{X} \rightarrow_{2} \underline{Y}, \underline{X} \rightarrow_{1} \bar{Y}\right]\right. \\
& =\left[\bar{Z} \rightarrow_{2}\left(\bar{X} \rightarrow_{2} \underline{Y}\right), \underline{Z} \rightarrow_{1}\left(\underline{X} \rightarrow_{1} \bar{Y}\right)\right]
\end{aligned}
$$

Corollary 2. Given a $B L$-algebra $\left\langle L, \wedge, \vee, *, \rightarrow, 0_{L}, 1_{L}\right\rangle$, for the best interval representation of $\rightarrow$, for all $X, Y, Z \in I(L)$, the following properties remain valid:

(A-1) If $X \sqsubseteq Y$ then $X \widehat{*} Z \sqsubseteq Y \widehat{*} Z$;

$(A-2) X \widehat{*} \mathbf{0}=\mathbf{0}$, where $\mathbf{0}=\left[0_{L}, 0_{L}\right]$;

(A-3) If $Y \sqsubseteq Z$ then $X \widehat{\rightarrow} Y \sqsubseteq X \widehat{\rightarrow} Z$ (Second Place Isotonicity);

$(A-4) X \widehat{\rightarrow}(Y \widehat{\rightarrow} Z)=Y \widehat{\rightarrow}(X \widehat{\rightarrow} Z)($ Exchange Principle $)$;

(A-5) If $X \sqsubseteq Y$ then $Y \widehat{\rightarrow} Z \sqsubseteq X \widehat{\rightarrow} Z$ (First Place Antitonicity);

$(A-6)(X \widehat{\vee} Y) \widehat{*} Z=(X \widehat{*} Z) \widehat{\vee}(Y \widehat{*} Z)$;

$(A-7)(X \widehat{*} Z) \widehat{\rightarrow} Y=Z \widehat{\rightarrow}(X \widehat{\rightarrow} Y)$;

Proposition 6. Let $\left\langle L, \wedge, \vee, *_{1}, \rightarrow_{1}, 0_{L}, 1_{L}\right\rangle$ and $\left\langle L, \wedge, \vee, *_{2}, \rightarrow_{2}, 0_{L}, 1_{L}\right\rangle$ be BL-algebras such that $*_{1} \leq *_{2}$ and $\rightarrow_{2} \leq \rightarrow_{1}$. $\widehat{\rightarrow}_{1,2}$ does not satisfy the Order Property and also the rules of calculus (BL6), (BL14), (BL15), (BL16), (BL17) and (BL19).

Proof. Indeed, for Order Property, just consider $X=\left[0_{L}, 1_{L}\right]$ so we have $X \widehat{\widehat{1}_{1,2}} X=\left[0_{L}, 1_{L}\right] \neq\left[1_{L}, 1_{L}\right]$. For Property (BL6) just consider $X=\left[0_{L}, 1_{L}\right]$ and $Y=\left[0_{L}, 0_{L}\right]$. Then $X \widehat{* 1,2}\left(X \widehat{\rightarrow_{1,2}} Y\right)=\left[0_{L}, 1_{L}\right]$ but $\left[0_{L}, 1_{L}\right] \sqsubseteq\left[0_{L}, 0_{L}\right]$ is not true . As for the property (BL14) just consider $X=\left[1_{L}, 1_{L}\right], Y=\left[0_{L}, 1_{L}\right]$ and $Z=\left[0_{L}, 0_{L}\right]$. Then $\left(X \widehat{\rightarrow 1,2}_{1, \widehat{*_{1,2}}}\left(Y \widehat{\rightarrow 1,2}_{1,2}\right)=\left[0_{L}, 1_{L}\right]\right.$, but $\left(X \widehat{\rightarrow}_{1,2} Z\right)=\left[0_{L}, 0_{L}\right]$. For property (BL15) just to make $X=\left[0_{L}, 1_{L}\right], Y=\left[1_{L}, 1_{L}\right]$ and $Z=\left[0_{L}, 1_{L}\right]$. Then $(X \widehat{\rightarrow 1,2} Y)=\left[1_{L}, 1_{L}\right]$, but

$$
\left(X \widehat{*_{1,2}} Z\right) \widehat{\rightarrow 1,2}\left(X \widehat{*_{1,2}} Y\right)=\left[0_{L}, 1_{L}\right] .
$$

For properties (BL17), (BL17) and (BL19) the checking follows easily making $X=\left[0_{L}, 1_{L}\right]$ and $Y=\left[1_{L}, 1_{L}\right]$. 
Corollary 3. The best interval representation of operator $\rightarrow$ does not satisfy the Order Property and also the rules of calculus (BL6), (BL14), (BL15), (BL16), (BL17) and (BL19). In what follows we show some properties that are satisfied by our proposed structure:

Theorem 3. Let $\left\langle L, \wedge, \vee, *_{1}, \rightarrow_{1}, 0_{L}, 1_{L}\right\rangle$ and $\left\langle L, \wedge, \vee, *_{2}, \rightarrow_{2}, 0_{L}, 1_{L}\right\rangle$ be BL-algebras such that $*_{1} \leq *_{2}$ and $\rightarrow_{2} \leq \rightarrow_{1}$. $\widehat{\rightarrow 1,2}_{1,}$ satisfies, for all $X, Y, Z \in I(L)$ and for all $x, y, z \in L$, the following properties:

(A8) If $\bar{X} \leq \bar{Y}$ then $X \widehat{*_{1,2}}\left(X \widehat{\rightarrow_{1,2}} Y\right) \sqsubseteq Y$;

(A9) If $\bar{X} \leq \underline{Y}$ then $X \widehat{\rightarrow_{1,2}} Y=\left[1_{L}, 1_{L}\right]$ ( $r$-Weak Order Property);

(A10) If $X \widehat{\rightarrow}_{1,2} Y=\left[1_{L}, 1_{L}\right]$ then $X \sqsubseteq Y$ ( $\ell$-Order Property);

(A11) If $\underline{X} \leq \underline{Y}$ then $\left(X \widehat{\rightarrow_{1,2}} Y\right) \widehat{*_{1,2}}(Y \widehat{\rightarrow 1,2} Z) \sqsubseteq X \widehat{\rightarrow_{1,2}} Z$;

(A12) If $Z$ is degenerate then $X \widehat{\rightarrow_{1,2}} Y \sqsubseteq\left(X \widehat{*_{1,2}} Z\right) \widehat{\rightarrow_{1,2}}\left(Z \widehat{*_{1,2}} Y\right)$;

(A13) If $\bar{Y} \leq \underline{X}$ then $(X \widehat{\wedge} Y) \widehat{\rightarrow 1,2} X=\mathbf{1}$, where $\mathbf{1}=\left[1_{L}, 1_{L}\right]$.

(A14) If $Y \sqsubseteq X$ then $X \widehat{\rightarrow 1,2} Y=X \widehat{\rightarrow 1,2}(X \widehat{\wedge} Y)$;

(A15) If $Y$ is degenerate then $X \sqsubseteq Y \widehat{\rightarrow}_{1,2}(Y \widehat{*} X)$.

Proof. The properties of operator presented in Section 1 are related with the respective properties of the best interval operator enrolled above.

(A8): Initially we have

$$
X \widehat{*_{1,2}}\left(X \widehat{\rightarrow_{1,2}} Y\right)=[\underline{X}, \bar{X}] \widehat{*_{1,2}}\left([\underline{X}, \bar{X}] \widehat{\rightarrow_{1,2}}[\underline{Y}, \bar{Y}]\right)=\left[\underline{X} *_{1}\left(\bar{X} \rightarrow_{2} \underline{Y}\right), \bar{X} *_{2}\left(\underline{X} \rightarrow_{1} \bar{Y}\right)\right] .
$$

On the other hand, since by (BL10) hold: (I) $\bar{X} \rightarrow_{2} \underline{Y} \leq \underline{X} \rightarrow_{2} \underline{Y} \stackrel{\text { (hyp.) }}{\leq} \underline{X} \rightarrow_{1} \underline{Y}$ and (II) $\bar{X} \rightarrow_{2}$ $\bar{Y} \stackrel{(\text { hyp. })}{\leq} \bar{X} \rightarrow_{1} \bar{Y} \leq \underline{X} \rightarrow_{1} \bar{Y}$. Hence by (BL7) in (I):

$$
\underline{X} *_{1}\left(\bar{X} \rightarrow_{2} \underline{Y}\right) \leq \underline{X} *_{1}\left(\underline{X} \rightarrow_{1} \underline{Y}\right) \stackrel{(B L 6)}{\leq} \underline{Y} .
$$

Further since $\bar{X} \leq \bar{Y}$, in (II) we have: $\bar{X} *_{2}\left(\underline{X} \rightarrow_{1} \bar{Y}\right)=\bar{X} *_{2} 1_{L}=\bar{X} \leq \bar{Y}$. Of these inequalities follows the result.

(A9): Since $\bar{X} \leq \underline{Y}$ then holds the follows inequalities

$$
\underline{X} \leq \bar{X} \leq \underline{Y} \leq \bar{Y}
$$

Thus, by property (BL11) follows the result. 
(A10): Also follows by property (BL11) easily.

(A11): Since $\underline{X} \leq \underline{Y}$, then the following statements are true

1. $\underline{Y} \rightarrow_{1} \bar{Y} \leq \underline{X} \rightarrow_{1} \bar{Y}$, por (BL9). Hence we conclude that $\underline{X} \rightarrow_{1} \bar{Y}=1$ since $\underline{Y} \rightarrow_{1} \bar{Y}=1$ and $\underline{X} \rightarrow_{1} \bar{Y} \leq 1$.

2. $\underline{Y} \rightarrow_{1} \bar{Z} \leq \underline{X} \rightarrow_{1} \bar{Z}$.

From these statements we obtain the expression

$$
(\underline{X} \rightarrow \bar{Y}) *(\underline{Y} \rightarrow \bar{Z})=1 *(\underline{Y} \rightarrow \bar{Z}) \leq \underline{X} \rightarrow \bar{Z} .
$$

Moreover, as $\underline{Y} \leq \bar{Y}$, by (BL10) we have $\bar{X} \rightarrow \underline{Y} \leq \bar{X} \rightarrow \bar{Y}$ and therefore

$$
(\bar{X} \rightarrow \underline{Y}) *(\bar{Y} \rightarrow \underline{Z}) \stackrel{(B L 7)}{\leq}(\bar{X} \rightarrow \bar{Y}) *(\bar{Y} \rightarrow \underline{Z}) \stackrel{(B L 14)}{\leq} \bar{X} \rightarrow \underline{Z}
$$

which allows us to conclude that

$$
(\bar{X} \rightarrow \underline{Y}) *(\bar{Y} \rightarrow \underline{Z}) \leq \bar{X} \rightarrow \underline{Z} .
$$

From expressions (3.1) and (3.2) it follows that

$$
\left[\left(\bar{X} \rightarrow_{2} \underline{Y}\right) *_{1}\left(\bar{Y} \rightarrow_{2} \underline{Z}\right),\left(\underline{X} \rightarrow_{1} \bar{Y}\right) *_{2}\left(\underline{Y} \rightarrow_{1} \bar{Z}\right)\right] \sqsubseteq\left[\bar{X} \rightarrow_{2} \underline{Z}, \underline{X} \rightarrow_{1} \bar{Z}\right] .
$$

(A12): Since $Z$ is degenerate, the result follows directly by applying the property (BL15).

(A13): Just see that $\bar{X} \wedge \bar{Y}=\bar{X} *_{2}\left(\bar{X} \rightarrow_{2} \bar{Y}\right) \leq \bar{Y} \leq \underline{X}$. So $\bar{X} \wedge \bar{Y} \rightarrow_{2} \underline{X}=1_{L}$ by (BL11). Analogously $\underline{X} \wedge \underline{Y}=\underline{X} *_{1}\left(\underline{X} \rightarrow_{1} \underline{Y}\right) \leq \underline{Y} \leq \bar{Y} \leq \underline{X} \leq \bar{X}$. Therefore $\underline{X} \wedge \underline{Y} \rightarrow_{1} \bar{X}=1_{L}$ by (BL11).

(A14): If $Y \sqsubseteq X$ then we can note the following facts

1. $\underline{Y} \leq \underline{X} \stackrel{(B L 11)}{\Leftrightarrow} \underline{Y} \rightarrow_{2} \underline{X}=1_{L} \stackrel{(B L 17)}{\Rightarrow} 1_{L}=\underline{Y} \rightarrow_{2}(\underline{X} \wedge \underline{Y}) \stackrel{(B L 11)}{\Leftrightarrow} \underline{Y} \leq(\underline{X} \wedge \underline{Y})$. Thus, by (BL6) follows that

$$
\bar{X} *_{2}\left(\bar{X} \rightarrow_{2} \underline{Y}\right) \leq(\underline{X} \wedge \underline{Y}) \stackrel{(B L 3)}{\Leftrightarrow} \bar{X} \rightarrow_{2} \underline{Y} \leq \bar{X} \rightarrow_{2}(\underline{X} \wedge \underline{Y}) .
$$

On the other hand, property (BL16) ensures that $\underline{X} \wedge \underline{Y} \rightarrow_{2} \underline{Y}=1_{L} \stackrel{(B L 11)}{\Leftrightarrow} \underline{X} \wedge \underline{Y} \leq \underline{Y}$. Then by (BL9) we have

$$
\bar{X} \rightarrow_{2}(\underline{X} \wedge \underline{Y}) \leq \bar{X} \rightarrow_{2} \underline{Y} .
$$

From (3.3) and (3.4) we get equality

$$
\bar{X} \rightarrow_{2} \underline{Y}=\bar{X} \rightarrow_{2}(\underline{X} \wedge \underline{Y}) .
$$


2. $\bar{Y} \leq \bar{X} \stackrel{(B L 11)}{\Leftrightarrow} \bar{Y} \rightarrow_{1} \bar{X}=1_{L} \stackrel{(B L 17)}{\Rightarrow} 1_{L}=\bar{Y} \rightarrow_{1}(\bar{X} \wedge \bar{Y}) \stackrel{(B L 11)}{\Leftrightarrow} \bar{Y} \leq(\bar{X} \wedge \bar{Y})$. Thus by (BL6) again follows that

$$
\underline{X} *_{1}\left(\underline{X} \rightarrow_{1} \bar{Y}\right) \leq(\bar{X} \wedge \bar{Y}) \stackrel{(B L 3)}{\Leftrightarrow} \underline{X} \rightarrow_{1} \bar{Y} \leq \underline{X} \rightarrow_{1}(\bar{X} \wedge \bar{Y}) .
$$

On the other hand, property (BL16) ensures that $\bar{X} \wedge \bar{Y} \rightarrow_{1} \bar{Y}=1_{L} \stackrel{(B L 11)}{\Leftrightarrow} \bar{X} \wedge \bar{Y} \leq \bar{Y}$. Then by (BL9) we have

$$
\underline{X} \rightarrow_{1}(\bar{X} \wedge \bar{Y}) \leq \underline{X} \rightarrow_{1} \bar{Y} .
$$

From (3.6) and (3.7) we get equality

$$
\underline{X} \rightarrow_{1} \bar{Y}=\underline{X} \rightarrow_{1}(\bar{X} \wedge \bar{Y}) .
$$

The equations (3.5) and (3.8) provide the desired result.

(A15) Since $Y$ is degenerate, the result follows directly by applying the property (BL15).

Corollary 4. The best interval representation of operator $\rightarrow$ satisfies, for all $X, Y, Z \in I(L)$ and for all $x, y, z \in L$, the following properties:

(A8) If $\bar{X} \leq \bar{Y}$ then $X \widehat{*}(X \widehat{\rightarrow} Y) \sqsubseteq Y$;

(A9) If $\bar{X} \leq \underline{Y}$ then $X \widehat{\rightarrow} Y=\left[1_{L}, 1_{L}\right]$ ( $r$-Weak Order Property);

(A10) If $X \widehat{\rightarrow} Y=\left[1_{L}, 1_{L}\right]$ then $X \sqsubseteq Y$ ( $\ell$-Order Property);

(A11) If $\underline{X} \leq \underline{Y}$ then $(X \widehat{\rightarrow} Y) \widehat{*}(Y \widehat{\rightarrow} Z) \sqsubseteq X \widehat{\rightarrow} Z$;

(A12) If If $Z$ is degenerate then $X \widehat{\rightarrow} Y \sqsubseteq(X \widehat{*} Z) \widehat{\rightarrow}(Z \widehat{*} Y)$;

(A13) If $\bar{Y} \leq \underline{X}$ then $(X \widehat{\wedge} Y) \widehat{\rightarrow} X=\mathbf{1}$, where $\mathbf{1}=\left[1_{L}, 1_{L}\right]$.

(A14) If $Y \sqsubseteq X$ then $X \widehat{\rightarrow} Y=X \widehat{\rightarrow}(X \widehat{\wedge} Y)$;

(A15) If If $Y$ is degenerate then $X \sqsubseteq Y \widehat{\rightarrow}(Y \widehat{*} X)$;

(A16) $[x, x] \widehat{\rightarrow}[y, y]=[z, z]$ (Degenerate Preservation).

\section{FINAL REMARKS}

This paper showed that it is impossible to have interval BL-algebras with all operations being correct. Hence a new abstract algebraic structure must be provided in order to model interval fuzzy logics. We showed how we could build an algebraic structure (whose elements are intervals) by starting from BL-algebras as well as revealed some of its properties. This provides a hint of how a new abstract algebraic structure must be in order to model both: BL-algebras and its interval counterpart. For further works, we aim to investigate how such structure could be and how would be entities like filters and ideals. 
RESUMO. Lógica Fuzzy Intervalar e Conjuntos Fuzzy valorados em intervalos têm sido amplamente investigado. Algumas Lógicas Fuzzy foram algebricamente modeladas por Peter Hájek como BL-álgebras. Qual é a contrapartida algébrica para o caso intervalar? Sabe-se da literatura que existe uma incompatibilidade entre algumas estruturas algébricas e sua contraparte intervalar. Este artigo mostra que tal incompatibilidade também está presente ao nível de BL-álgebras. Aqui mostramos ambos: (1) a impossibilidade na imprecisão correspondente e a corretude da fundamental BL-Implicação e (2) alguns fatos sobre a intervalização de BL-álgebras.

Palavras-chave: Lógica Fuzzy, BL-Álgebras, intervalos, princípio de corretude.

\section{REFERENCES}

[1] P. Aglianó. Varieties of BL-Algebras III: Splitting Algebras. Studia Logica, (2018). doi:10.1007/ s11225-018-9836-2. URL https://doi .org/10.1007/s11225-018-9836-2.

[2] P. Agliano \& F. Montagna. Varieties of BL-algebras I: general properties. Journal of Pure and Applied Algebra, 181(2) (2003), 105-129. doi:https://doi.org/10.1016/S0022-4049(02)00329-8. URL http: //www.sciencedirect.com/science/article/pii/S0022404902003298.

[3] P. Aglianò \& F. Montagna. Varieties of BL-Algebras II. Studia Logica, 106(4) (2018), 721-737. doi: 10.1007/s11225-017-9763-7. URL https://doi .org/10.1007/s11225-017-9763-7.

[4] B.C. Bedregal \& R.H.N. Santiago. Interval representations, Łukasiewicz implicators and Smets-Magrez axioms. Information Sciences, 221 (2013), 192-200. doi:http://dx.doi.org/10. 1016/j.ins.2012.09.022. URL http://www.sciencedirect.com/science/article/pii/ S0020025512006159.

[5] H. Bustince. Interval-valued Fuzzy Sets in Soft Computing. International Journal of Computational Intelligence Systems, 3(2) (2010), 215-222. doi:10.1080/18756891.2010.9727692. URL http: //dx . doi.org/10.1080/18756891.2010.9727692.

[6] L.M. Cabrer \& D. Mundici. Interval MV-algebras and generalizations. International Journal of Approximate Reasoning, 55(8) (2014), 1623-1642. doi:http://dx.doi.org/10.1016/j.ijar.2014.05.002. URL http://www. sciencedirect. com/science/article/pii/S0888613X14000814.

[7] A. Di Nola \& L. Leuştean. Compact representations of BL-algebras. Archive for Mathematical Logic, 42(8) (2003), 737-761. doi:10.1007/s00153-003-0178-y. URL http://dx.doi.org/10. 1007/s00153-003-0178-y.

[8] A. Di Nola, S. Sessa, F. Esteva, L. Godo \& P. Garcia. The Variety Generated by Perfect BL-Algebras: an Algebraic Approach in a Fuzzy Logic Setting. Annals of Mathematics and Artificial Intelligence, 35(1) (2002), 197-214. doi:10.1023/A:1014539401842. URL http://dx.doi.org/10.1023/A: 1014539401842.

[9] P. Hajek. "Metamathematics of Fuzzy Logic". Kluwer Academic Publishers (1998).

[10] T. Hickey, Q. Ju \& M.H. Van Emden. Interval Arithmetic: From Principles to Implementation. J. ACM, 48(5) (2001), 1038-1068. doi:10.1145/502102.502106. URL http://doi . acm.org/10. $1145 / 502102.502106$. 
[11] R.B. Kearfott \& V. Kreinovich (editors). "Applications of Interval Computations: An Introduction". Springer US, Boston, MA (1996), pp. 1-22. doi:10.1007/978-1-4613-3440-8_1. URL http://dx. doi.org/10.1007/978-1-4613-3440-8_1.

[12] R.E. Moore \& F. Bierbaum. "Methods and Applications of Interval Analysis (SIAM Studies in Applied and Numerical Mathematics) (Siam Studies in Applied Mathematics, 2.)". Soc for Industrial \& Applied Math (1979).

[13] S. Motamed \& L. Torkzadeh. A new class of BL-algebras. Soft Computing, 21(3) (2017), 687-698. doi:10.1007/s00500-016-2043-z. URL https ://doi .org/10.1007/s00500-016-2043-z.

[14] R.H.N. Santiago, B. Bedregal, A. Madeira \& M.A. Martins. On Interval Dynamic Logic. In L. Ribeiro $\&$ T. Lecomte (editors), "Formal Methods: Foundations and Applications". Springer International Publishing, Cham (2016), pp. 129-144.

[15] R.H.N. Santiago, B.R.C. Bedregal \& B.M. Acióly. Formal Aspects of Correctness and Optimality of Interval Computations. Formal Aspects of Computing, 18(2) (2006), 231-243. doi:10.1007/ s00165-006-0089-x. URL http://dx.doi.org/10.1007/s00165-006-0089-x.

[16] B. Van Gasse, C. Cornelis \& G. Deschrijver. Interval-valued algebras and fuzzy logics. In C. Cornelis, G. Deschrijver, M. Nachtegael, S. Schockaert \& Y. Shi (editors), "35 Years of fuzzy set theory: celebratory volume dedicated to the retirement of Etienne E. Kerre", volume 261 of Studies in Fuzziness and Soft Computing. Springer (2011), pp. 57-82. URL http://dx.doi.org/10.1007/ 978-3-642-16629-7_4.

[17] J. Yang, X.L. Xin \& P.F. He. Notes on topological BL-algebras. Fuzzy Sets and Systems, 350 (2018), 33-40. doi:https://doi.org/10.1016/j.fss.2017.10.011. URL http://www . sciencedirect. com/science/article/pii/S0165011417303822. 